\title{
Viscosimetric analysis of the mechanism of ATP hydrolysis by pea chloroplast $\mathbf{F}_{1}$-ATPase
}

\author{
A.N. MALYAN \\ Institute of Basic Biological Problems, Russian Academy of Sciences, Institutskaya 2, 142290, Pushchino, \\ Moscow Region, Russia
}

\begin{abstract}
Dependence of ATP hydrolysis kinetics by the chloroplast coupling factor $\left(\mathrm{CF}_{1}\right)$ on medium viscosity was studied at varying temperatures. For samples with oxidized and reduced $\mathrm{CF}_{1} \gamma$-subunit, this dependence was shown to be described by Cramers' relationship $k \sim\left(\eta / \eta_{\mathrm{o}}\right)^{-n}$, where $k$ is the reaction rate constant, $\eta / \eta_{\mathrm{o}}$ is the medium/water viscosity ratio, and $0<n<1$. Transition of the $\gamma$-subunit from its reduced to oxidized state was accompanied by increasing $n$ value, which is indicative of increasing friction losses between certain enzyme sections and the solution. The increased medium viscosity produced no effect on the reaction activation energy which appeared to be almost the same for the both enzyme states. The molecular mechanisms responsible for $\mathrm{CF}_{1}$ activity loss in viscous media are discussed.
\end{abstract}

Additional keywords: ATP hydrolysis; F1-ATPase; kinetics; viscosity.

\section{Introduction}

The chloroplast $\mathrm{F}_{1}$-ATPase $\left(\mathrm{CF}_{1}\right)$ is a stroma-exposed catalytic part of the enzymatic complex $\mathrm{CF}_{0} \mathrm{~F}_{1}$ that synthesizes ATP from ADP and phosphate using energy of electrochemical transmembrane potential of protons. $\mathrm{CF}_{1}$ extracted into aqueous phase exhibits ATPase activity. The aqueous medium typically used for finding kinetic parameters of ATP synthesis/hydrolysis has a viscosity close to that of water $\left(1 \mathrm{cP}\right.$ at $\left.20^{\circ} \mathrm{C}\right)$. According to indirect estimates, chloroplast stroma viscosity is much higher (Ellis 1979, Asada 2004, Wayne 2009), which may result in diffusion-caused inhibition of the reaction, thereby making its in vivo kinetic parameters differ much from those reported in the literature (Kartashov et al. 2015, Malyan 2016). Also, as exemplified by a number of enzymes, high viscosity is capable of suppressing enzyme activity, provided that a part of energy required for conformational changes of an enzyme molecule during catalysis is spent on medium friction (Kramers 1940, Puchkov 2013). Therefore, knowledge of the viscosity effect would not only elucidate the difference between kinetic parameters of the reaction in question in vitro and in vivo but also shed light on the catalytic mechanism involved. This issue is of special interest because the functional mechanisms of chloroplast, bacterial, and mitochondrial $F_{1}$-ATPases differ dramatically from enzymes so far subjected to the viscosimetric analysis. According to modern concepts, $\mathrm{F}_{1}$-ATPases may be regarded as rotary engines, where chemical energy released during ATP hydrolysis at catalytic sites located at the interface between alternating $3 \alpha$ - and $3 \beta$-subunits, which act as a stator, turns into mechanical energy of rotation of $\gamma$ - and $\varepsilon$-subunits (for the $\mathrm{F}_{1}$-ATPase structure, see, e.g., review of Nakanishi-Matsui et al. (2016). The rotation is interrupted by dwells corresponding to the steps of substrate binding, its catalytic conversion, and dissociation of reaction products (Noji et al. 2017). In the course of ATP hydrolysis, $\mathrm{F}_{1}$-ATPases undergo considerable conformational changes including the mentioned rotation of $\gamma$ - and $\varepsilon$-subunits, an essential rearrangement of the active site domain, and reciprocal motions of the $\beta$ subunit C-terminus (Abrahams et al. 1994, NakanishiMatsui et al. 2016). The issue of the efficiency of $\mathrm{F}_{1}$ ATPase-induced energy conversion is currently in the focus of discussion in the literature. Some authors

Received 1 December 2017, accepted 9 March 2018, published as online-first 21 June 2018.

E-mail: alexander.malyan@gmail.com

Abbreviations: $\mathrm{CF}_{1}$ - chloroplast coupling factor $1 ; \mathrm{CF}_{\text {ox }}$ and $\mathrm{CF}_{\text {red }}-\mathrm{CF}_{1}$ preparations with oxidized or reduced $\mathrm{C} 199-\mathrm{C} 205$ bridge at the $\gamma$-subunit; $E$ - activation energy, $\mathrm{kcal} \mathrm{mol}^{-1} ; k$ - reaction rate constant; $K_{\mathrm{m}}$ - apparent Michaelis constant; MgATP - equimolecular mixture of $\mathrm{MgCl}_{2}$ with ATP; $V_{\max }$ - maximum reaction rate; $\mathrm{T}$ - absolute temperature in ${ }^{\circ} \mathrm{K} ; \eta$ and $\eta_{\mathrm{o}}$ - viscosity values of solution and solvent in $\mathrm{cP}$.

Acknowledgements: The author thanks M. Shkunova for help in some experiments and E.V. Serebrova for assistance in manuscript preparation. 
conclude that they are 100\% efficient (Yasuda et al. 1998, Kinosita et al. 2000, Itoh et al. 2004, Toyabe et al. 2011, Soga et al. 2017), while others point to drawbacks in interpretation of the experimental data, thus evaluating the $\mathrm{F}_{1}$-ATPase functioning efficiency lower than 100\% (Martin et al. 2014, Chapman and Loiselle 2016, Kulish et al. 2016). The theoretical studies by Kramers and others provide the basis for a qualitative estimate of energy loss resulting from protein-medium interaction in the course of reaction-induced conformational rearrangements of the protein molecule using the empirical coefficient $n$ from the relationship:

$$
k \sim\left(\eta / \eta_{\mathrm{o}}\right)^{-n}
$$

where $k$ is the reaction rate constant, $\eta$ and $\eta_{\mathrm{o}}$ are viscosity values of a solution and solvent, respectively, $0<n<1$ (Kramers 1940, Sitnitsky 2010). The larger $n$, the higher the inhibitory effect of medium viscosity on protein conformational rearrangements (Uribe and Sampedro 2003, Barbier and Campbell 2005). According to this theory, the activity decrease observed in viscous solutions results from increasing frictional losses between sites of the enzyme undergoing conformational rearrangements and molecules of the medium, which lead to an increase in the activation energy of the reaction (Khoshtariya and Goguadze 1986). However, it should be noted that recent studies of viscosity dependence of enzyme reaction rates give grounds for questioning the universality of the relationship (1) and the mentioned limits of variation of the viscosity exponent. Sashi and Bhuyan (2015) report that this dependence is determined by many interdependent factors, including both mode and consequences of proteinsolvent interactions. To study the viscosity dependence of enzyme reaction kinetics, substances modulating viscosity of aqueous solutions (viscosigens) are used. Singlemolecule measurements of F1-ATPase in E. coli (Spatzler et al. 2009) revealed that a polyethylene glycol (PEG400)-induced increase of medium viscosity lengthens dwells in $\gamma$-subunit rotation coupled with the stages of ATP binding and conversion. With polyethylene glycol, dextran, and sucrose acting as viscosigens, we found that

\section{Materials and methods}

The coupling factor $\mathrm{CF}_{1}$ was isolated from pea chloroplasts according to Binder et al. (1978) and stored in $2 \mathrm{M}$ ammonium sulfate in the presence of $1 \mathrm{mM}$ ATP, $1 \mathrm{mM}$ EDTA, and $50 \mathrm{mM}$ Tris- $\mathrm{SO}_{4}, \mathrm{pH}$ 7.8. Excess nucleotides and ammonium sulfate were removed by gel filtration through a Fine Sephadex G-50 column equilibrated with $50 \mathrm{mM}$ Tricine-KOH, $\mathrm{pH}$ 7.8. To unmask ATPase activity, $\mathrm{CF}_{1}$ was incubated with $50 \mathrm{mM}$ dithiothreitol for $2 \mathrm{~h}$ at room temperature. Protein concentration was determined according to Bradford (1976). The ATP hydrolysis rate was determined by HPLC. For this purpose, $15 \mu 1$ reaction mixture was loaded onto a $0.5 \times 7.5$ DEAE $5 \mathrm{PW}$ column their limited concentrations increased Michaelis constant of the photophosphorylation reaction without affecting its maximum rate, which was indicative of the diffusion nature of reaction inhibition (Kartashov et al. 2015). Further enhanced viscosigen concentrations with accordingly increased medium viscosity gave lower maximum rates of photophosphorylation, but interpretation of these results was impeded by the evolving uncoupling and inhibition of electron transport, as well as structural heterogeneity of the chloroplasts. These difficulties could be avoided when using homogeneous aqueous solutions of the coupling factor $\mathrm{CF}_{1}$ exhibiting ATPase activity. ATPase properties of $\mathrm{CF}_{1}$ from spinach chloroplasts were maintained by its latent-to-active state transition due to reduction of the disulfide bridge (C199-C205) in the $\gamma$-subunit (Nalin and McCarty 1984) and addition of sulfite and ethanol to the reaction medium [for details see Malyan (2016) and references therein]. Similar to the viscosity influence on the photophosphorylation rate, the Michaelis constant was observed to grow after sucrose addition to the medium, which is indicative of a diffusion character of reaction inhibition at low substrate concentrations. It was also found that the use of an enzyme containing oxidized dithiol (C199-C205) resulted in a different kind of dependence, that is, with decreasing maximum reaction rate, the Michaelis constant remained virtually unchanged. The current study was aimed to elucidate the mechanisms responsible for altered kinetics of ATP hydrolysis in viscous reaction mixtures over a wide range of viscosity and temperature. In experiment, we used pea chloroplast coupling factor displaying a high ATPase activity. For samples with oxidized and reduced $\gamma$-subunit, further referred to as $\mathrm{CF}_{\text {ox }}$ and $\mathrm{CF}_{\text {red }}$, the obtained dependencies were shown to be described by the relationship (1); importantly, the exponential factor was higher for $\mathrm{CF}_{\mathrm{ox}}$. An increase in medium viscosity produced no effect on the activation energy of reaction catalyzed by either form of the coupling factor; the activation energy values of the both forms were found to be close to each other. The mechanisms responsible for $\mathrm{CF}_{1}$-ATPase activity loss in viscous media are discussed.

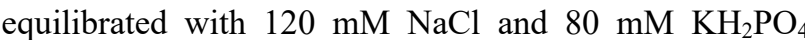
( $\mathrm{pH} 4.0$ ), followed by calculation of ADP and ATP amounts from the appropriate peaks. The reaction was performed in a constant-temperature vessel with magnetic stirring at $25^{\circ} \mathrm{C}$ in $0.5 \mathrm{ml}$ of the reaction mixture containing $50 \mathrm{mM}$ Tricine- $\mathrm{KOH}$ (pH 7.8), $0.3 \mathrm{mM} \mathrm{MgCl}_{2}, 75 \mathrm{mM}$ $\mathrm{K}_{2} \mathrm{SO}_{3}, 10 \%$ (v) $\mathrm{C}_{2} \mathrm{H}_{5} \mathrm{OH}, 1.5-3.0 \mu \mathrm{g}\left(\mathrm{CF}_{1}\right) \mathrm{ml}^{-1}, \mathrm{MgATP}$ and sucrose concentrations as indicated in the figures. Incubation time (5-10 $\mathrm{min}$ ) and protein concentrations were chosen so that ATP conversion did not exceed 10\%. Equimolecular mixture of $\mathrm{MgCl}_{2}$ with ATP was used as a reaction substrate. The match between viscosity and mass 
fraction of sucrose was determined using reference data (Asadi 2005). Graph approximation and calculation of

\section{Results}

In order to elucidate the viscosity effect on kinetic parameters of ATP hydrolysis, changes in the maximum reaction rate ( $V_{\max }$, showing enzymatic activity) and in the apparent Michaelis constant $\left(K_{\mathrm{m}}\right)$ were estimated upon addition of sucrose to the reaction mixture. An analysis of dependencies shown in Fig. 1 revealed that with $31 \%$ sucrose concentration corresponding to viscosity of $3.0 \mathrm{cP}$ (Asadi 2005), $V_{\max }$ of ATP hydrolysis by $\mathrm{CF}_{\text {red }}$ decreased from $0.34 \pm 0.01$ to $0.23 \pm 0.01 \mu \mathrm{mol} \mathrm{s}^{-1} \mathrm{mg}^{-1}$ (protein). The value of $K_{\mathrm{m}}$ increased from $0.57 \pm 0.07$ to $0.79 \pm 0.07 \mathrm{mM}$. The $\mathrm{CF}_{\text {ox }}$ samples showed a lower maximum reaction rate $\left(0.22 \pm 0.01 \mu \mathrm{mol} \mathrm{s}^{-1} \mathrm{mg}^{-1}\right)$ that further decreased to $0.13 \pm$ $0.008 \mu \mathrm{mol} \mathrm{s}^{-1} \mathrm{mg}^{-1}$ after sucrose addition to the mixture, while $K_{\mathrm{m}}$ remained practically unchanged $(0.51 \pm$ $0.09 \mathrm{mM}$ ). To reduce the experimental error, hydrolysis rates obtained at MgATP concentrations exceeding the 5-8-fold $K_{\mathrm{m}}$ were taken in subsequent experiments as the maximum reaction rates.

Fig. $2 A$ shows sucrose concentration dependency of the rates of reactions catalyzed by $\mathrm{CF}_{\text {red }}$ and $\mathrm{CF}_{\text {ox }}$. For $\mathrm{CF}_{\text {red }}$ over a sucrose concentration range of $5-20 \%$, a decrease of the reaction rate never reached $10 \%$, thus corresponding to a moderate viscosity of the medium $(<1.7 \mathrm{cP})$. With sucrose concentration increased to $40 \%$, the viscosity level was much higher, thus causing an essential decrease in the reaction rate. This effect was more prominent in case of $\mathrm{CF}_{\text {ox }}$ as compared to $\mathrm{CF}_{\text {red. }}$ An analysis of the results presented in the $\ln V_{\max }-\ln \eta$ coordinates (Fig. $2 B$ ) shows that the dependency is described by the relationship (1); it is of note that the term $n$ is higher for $\mathrm{CF}_{\mathrm{ox}}$ than that for $\mathrm{CF}_{\text {red }}$ (0.54 and 0.34, respectively).

To address the issue of viscosity dependence of ATP

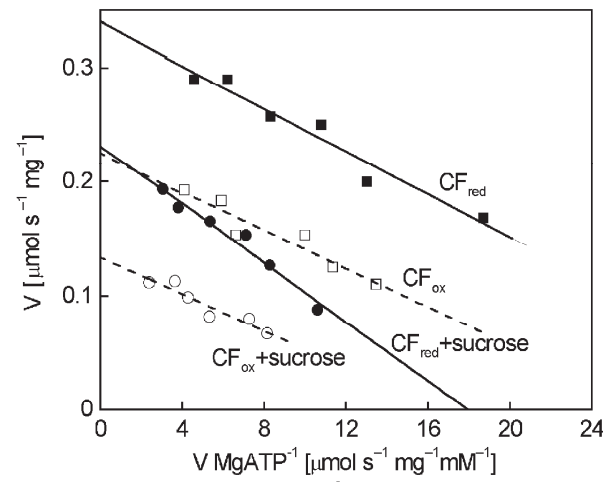

Fig. 1. Eadie-Hofstee graph presenting dependence of the ATP hydrolysis rate on MgATP concentration without or with $31 \%$ sucrose for reduced $\left(\mathrm{CF}_{\text {red }}\right)$ and oxidized $\left(\mathrm{CF}_{\text {ox }}\right)$ forms of $\mathrm{CF} 1$. $\mathrm{V}$ - reaction rate. For reaction conditions see Materials and methods section. kinetic parameters were performed using Origin 6.
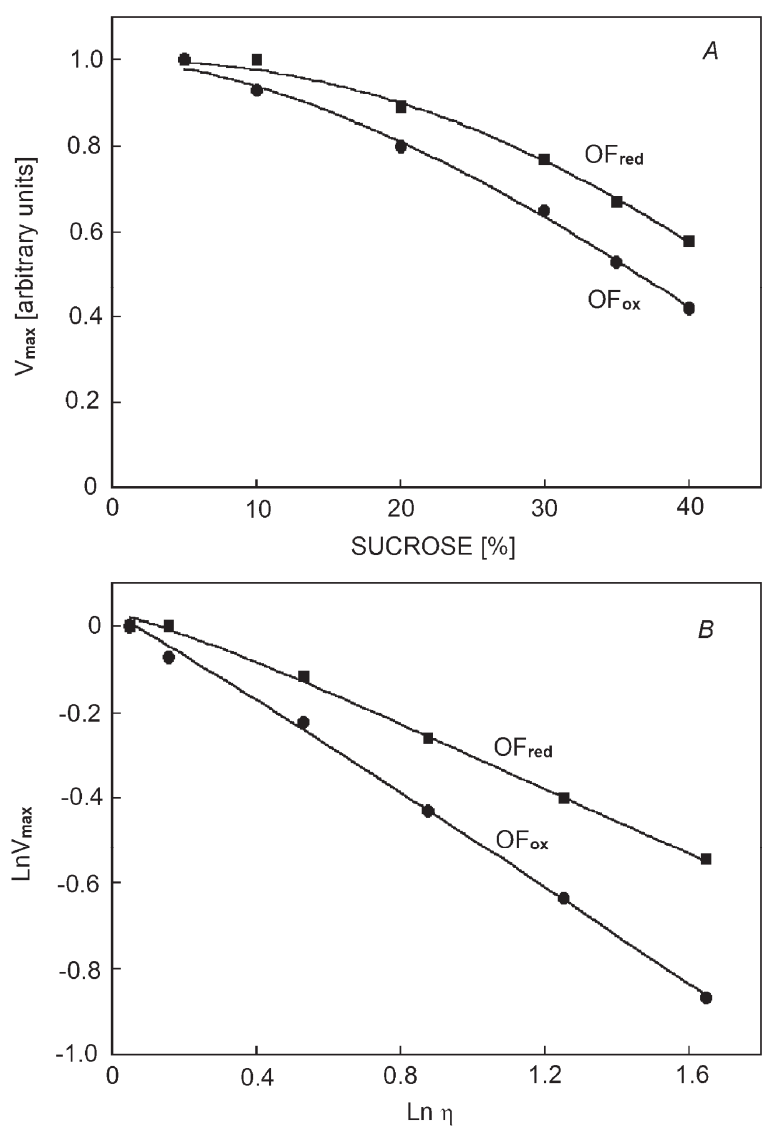

Fig. 2. Dependence of the maximum rate of ATP hydrolysis $\left(\mathrm{V}_{\max }\right)$ catalyzed by reduced/oxidized $\mathrm{CF} 1$ on $(A)$ sucrose concentration and $(B)$ medium viscosity (logarithmic coordinates). Reaction rates of $0.34 \mu \mathrm{mol} \mathrm{s}{ }^{-1} \mathrm{mg}^{-1}$ and $0.22 \mu \mathrm{mol} \mathrm{min} \mathrm{mg}^{-1}$ for $\mathrm{CF}_{\text {red }}$ and $\mathrm{CF}_{\mathrm{ox}}$, respectively, were taken to be equal to unity.

hydrolysis activation energy, temperature dependence of the reaction rate was studied (Fig. 3). The data from Asadi (2005) were used to select sucrose concentrations that allow unchanged viscosity of $3 \mathrm{cP}$ at increasing temperature. Activation energy $(E)$ calculated from the Arrhenius equation

$$
k=A e^{-E / R T}
$$

for linear sections of the curves in the absence and presence of sucrose amounted to 19.5 and $19.4 \mathrm{kcal} \mathrm{mol}^{-1}$, respectively, in case of $\mathrm{CF}_{\text {red }}$, and 20.1 and $19.3 \mathrm{kcal} \mathrm{mol}^{-1}$ in case of $\mathrm{CF}_{\text {ox., }}$ i.e., within the experimental error they remained practically constant. Thus, the viscosity level increasing within $3 \mathrm{cP}$ did not affect the activation energy, irrespective of the oxidized or reduced state of the $\mathrm{CF}_{1}$ $\gamma$-subunit. 


\section{Discussion}

The fact that the apparent Michaelis constant increased with increasing medium viscosity in experiments on reduced $\mathrm{CF}_{1}\left(\mathrm{CF}_{\text {red, }}\right.$ Fig. 1) is consistent with the results previously obtained for the similar enzyme from spinach chloroplasts (Malyan 2016) that can be explained by overcoming the diffusion effects by elevated MgATP concentrations. The activity decrease (reduced $V_{\max }$ ) as well as the value of $n=0.34$ are apparently caused by impeded structural rearrangements of the functioning enzyme in a viscous mixture and agree with the supposition (Martin et al. 2014, Chapman and Loiselle 2016, Kulish et al. 2016) that the $\mathrm{CF}_{1}$ effectiveness is below $100 \%$. According to modern concepts (Abrahams et al. 1994, Martin et al. 2014, Noji et al. 2017), ATP binding to the catalytic site of one of the $\beta$-subunits makes its C-terminal loop shift towards this site and push the $\gamma$-subunit, thus causing its rotation. Simultaneously, the C-terminal loop of another $\beta$-subunit moves in the opposite direction, which is accompanied by dissociation of reaction products. At the catalytic site of the third $\beta$-subunit ATP turns into ADP plus phosphate. Formally speaking, the absence of notable changes in activation energy under conditions of elevated viscosity (Fig. 3) indicates that enzymatic activity is reduced due to a lower pre-exponential factor in the Arrhenius equation which, in turn, might be provoked by longer dwells in $\gamma$-subunit rotation at the steps of substrate binding and converting in conditions of growing medium viscosity, similar to what was reported for $E$. coli $\mathrm{F}_{1}$-ATPase by Spetzler et al. (2009). According to the proposed interpretation of Kramer's theory (1940), the absence of activation energy changes suggests that conformational changes of the catalytic site domain at the step of conversion of tightly bound ATP into ADP and phosphate are unrestricted by medium viscosity. Then, the $\mathrm{CF}_{1}$-ATPase under study differs from both myosin ATPase and $\mathrm{E}_{1} \mathrm{E}_{2}$-ATPase of Kluyveromyces lactis plasma membrane whose activation energy increases with increasing medium viscosity (Khoshtariya and Goguadze 1986, Sampedro and Uribe 2004).

The absence of $K_{\mathrm{m}}$ alteration during the $\mathrm{CF}_{\mathrm{ox}}$-induced catalysis shows that, in contrast to $\mathrm{CF}_{\text {red }}$ catalysis, the reaction rate is unaffected by diffusion induced inhibition. This is a well-expected result because the $\mathrm{CF}_{\mathrm{ox}}$ activity is approximately two times lower than that of $\mathrm{CF}_{\text {red }}$ (Fig. 1). An increase of the empirical factor $n$ (Fig. 2B) may result from increased friction losses between certain enzyme sections and the solution. According to Konno et al. (2012), the oxidized and reduced states of the enzyme

\section{References}

Abrahams J.P., Leslie A.G., Lutter R., Walker J.E.: Structure at 2.8A resolution of F1-ATPase from bovine heart mitochondria. - Nature 370: 621-628, 1994.

Asada K.: Radical production and scavenging in the chloroplasts.

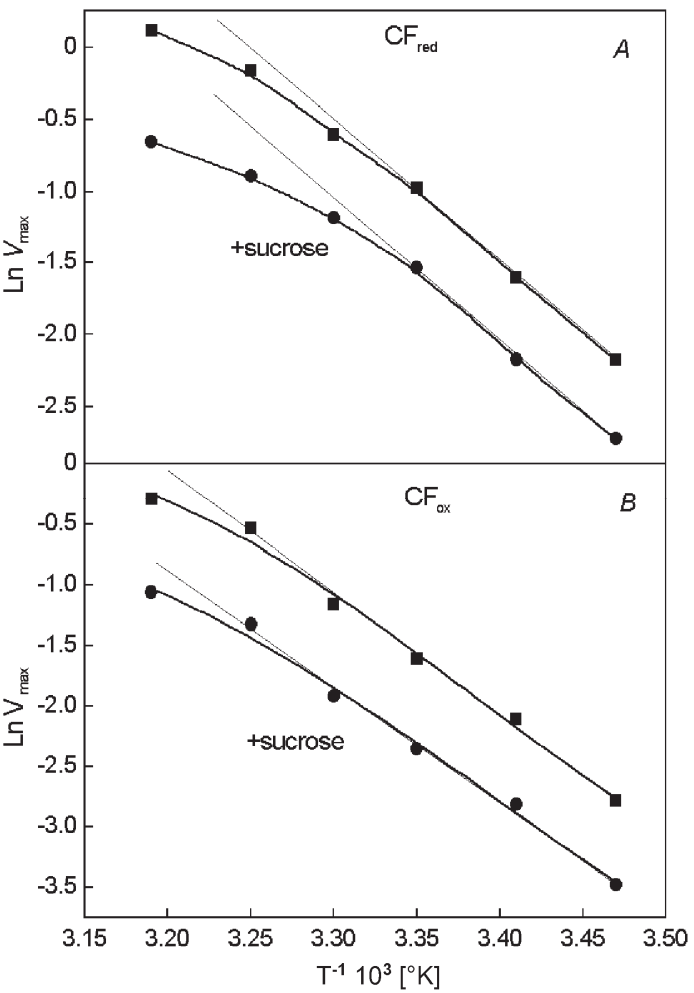

Fig. 3. Medium viscosity independent activation energy of ATP hydrolysis catalyzed by reduced $(A)$ /oxidized $(B)$ forms of CF 1 . The sucrose concentrations at each temperature that allow unchanged viscosity of $3 \mathrm{cP}$ selected from Table A12 of Asadi (2005) were as follows: $26 \%$ sucrose at $15^{\circ} \mathrm{C}, 29 \%$ at $20^{\circ} \mathrm{C}$, $31.5 \%$ at $25^{\circ} \mathrm{C}, 34 \%$ at $30^{\circ} \mathrm{C}, 36.5 \%$ at $35^{\circ} \mathrm{C}, 39 \%$ at $40^{\circ} \mathrm{C}$. T absolute temperature; $\mathrm{LnV}_{\max }$ - natural logarithm of the maximum reaction rate.

differ from each other by behavior of its $\varepsilon$-subunit. In $\mathrm{F}_{1}$-ATPases of different origins, the state of this subunit may be either "down" or "up", the latter meaning that the $\varepsilon$-subunit C-terminus is embedded between the stator $\alpha$-, $\beta$-subunits and the double $\alpha$-helix of the rotor $\gamma$-subunit, thus preventing rotation of this subunit and making the enzyme inactive (Konno et al. 2012, Nakanishi-Matsui et al. 2016). It was shown that frequency of down-to-up transitions of the $\varepsilon$-subunit and duration of its keeping the up state (which in turn determines frequency and duration of dwells in rotor rotation) increase after enzyme oxidation. This allows suggestion that an increase of the exponent $n$ in relationship (1) results from more frequent structural rearrangements of the $\varepsilon$-subunit, which is characteristic of the enzyme with oxidized $\gamma$-subunit.

- In: Baker N.R. (ed.): Photosynthesis and the Environment. Pp. 123-150. Kluwer Acad. Publ., Dordrecht - Boston London 2004.

Asadi M.: Beet-Sugar Handbook, Table A12, Viscosity of Pure 
Sucrose Solutions. Retrieved from: http://www.wiley.com/ doi/10.1002/9780471790990.cth1/pdf, 2005.

Barbier G.G., Campbell W.H.: Viscosity effects on eucaryotic nitrate reductase activity. - J. Biol. Chem. 280: 26049-26054, 2005.

Binder A., Jagendorf A., Ngo E.: Isolation and composition of the subunits of spinach chloroplast coupling factor protein. $-\mathrm{J}$. Biol. Chem. 253: 3094-3100, 1978.

Bradford M.M.: A rapid and sensitive method for the quantitation of microgram quantities of protein utilizing the principle of protein-dye binding. - Anal. Biochem. 72: 248-254, 1976.

Chapman B., Loiselle D.: Thermodynamics and kinetics of the FoF1 ATPase: application of the probability isotherm. - R. Soc. Open Sci. 3: 150379, 2016.

Ellis R.: The most abundant protein in the world. - Trends Biochem. Sci. 4: 241-244, 1979.

Itoh H., Takahashi A., Adachi K. et al.: Mechanically driven ATP synthesis by F1-ATPase. - Nature 427: 465-468, 2004.

Kartashov I.M., Opanasenko V.K., Malyan A.N.: [Effects of medium viscosity agents on ATP synthesis in chloroplast thylakoids.] - Biofizika 60: 481- 486, 2015. [In Russian]

Khoshtariya D.E., Goguadze N.G.: [Influence of the medium viscosity on the activity of myosin ATPase.] - Biofizika 31: 220-222, 1986. [In Russian]

Kinosita K. Jr., Yasuda R., Noji H., Adachi K.: A rotary molecular motor that can work at near $100 \%$ efficiency. Philos. T. Roy. Soc. B 355: 473-489, 2000.

Konno H., Nakane T., Yoshida M. et al.: Thiol modulation of the chloroplast ATP synthase is dependent on the energization.Plant Cell Physiol. 53: 626-634, 2012.

Kramers H.A.: Brownian motion in a field of force and the diffusion model of chemical reactions. - Physica 7: 284-304, 1940.

Kulish O., Wright A.D., Terentjev E.M.: F1 rotary motor of ATP synthase is driven by the torsionally asymmetric drive shaft. Sci. Rep. 6: 28180, 2016.

Malyan A.N.: The effect of medium viscosity on kinetics of ATP hydrolysis by the chloroplast coupling factor $\mathrm{CF}_{1}$. Photosynth. Res. 128: 163-168, 2016.

Martin J.L., Ishmukhametov R., Hornung T. et al.: Anatomy of F1-ATPase powered rotation. - P. Natl. Acad. Sci. USA 111:
3715-3720, 2014.

Nakanishi-Matsui M., Sekiya M., Futai M.: ATP synthase from Escherichia coli: Mechanism of rotational catalysis, and inhibition with the $\varepsilon$-subunit and phytopolyphenols. - Biochim. Biophys. Acta 1857: 129-140, 2016.

Nalin C.M., McCarty R.E.: Role of a disulfide bond in the gamma subunit in activation of the ATPase of chloroplast coupling fator 1. - J. Biol. Chem. 159: 7275-7280, 1984.

Noji H., Ueno H., McMillan D.G.G.: Catalytic robustness and torque generation of the F1-ATPase. - Biophys. Rev. 9: 103118, 2017.

Puchkov E.O.: Intracellular viscosity: methods of measurement and role in metabolism. - Biochemistry-Moscow + 7: 270-279, 2013.

Sampedro J., Uribe S.: Trehalose-enzyme interactions result in structure stabilization and activity inhibition. The role of viscosity. - Mol. Cell Biochem. 256-257: 319-327, 2004.

Sashi P., Bhuyan A.K.: Viscosity dependence of some protein and enzyme reaction rates: seventy-five years after Kramers. Biochemistry 54: 4453-4461, 2015.

Sitnitsky A.E.: Model for solvent viscosity effect on enzymatic reactions. - Chem.. Phys. 369: 37-42, 2010.

Soga N., Kimura K., Kinosita K.Jr. et al.: Perfect chemomechanical coupling of FoF1-ATP synthase. - P. Natl. Acad. Sci. USA 114: 4960-4965, 2017.

Spetzler D., Ishmukhametov R., Hornung T. et al.: Single molecule measurements of F1-ATPase reveal an interdependence between the power stroke and the dwell duration. Biochemistry 48: 7979-7985, 2009.

Toyabe S., Watanabe-Nakayama T., Okamoto T. et al.: Thermodynamic efficiency and mechanochemical coupling of F1ATPase. - P. Natl. Acad. Sci. USA 108: 17951-17956, 2011.

Uribe S., Sampedro J.G.: Measuring solution viscosity and its effect on enzyme activity. - Biol. Proced. Online 5: 108-115, 2003.

Wayne R.O.: Plant Cell Biology: from Astronomy to Zoology. Elsevier, Dordrecht - Boston - London 2009.

Yasuda R., Noji H., Kinosita K.Jr., Yoshida M.: F1-ATPase is a highly efficient molecular motor that rotates with discrete $120^{\circ}$ steps. - Cell 93: 1117-1124, 1998. 